\title{
Characteristic of nanoparticles generated from different nano-powders by using different dispersion methods
}

\author{
Chuen-Jinn Tsai · Guan-Yu Lin • \\ Chun-Nan Liu $\cdot$ Chi-En He $\cdot$ Chun-Wan Chen
}

Received: 14 November 2011/Accepted: 11 February 2012/Published online: 10 March 2012

(C) Springer Science+Business Media B.V. 2012

\begin{abstract}
A standard rotating drum with a modified sampling train (RD), a vortex shaker (VS), and a SSPD (small-scale powder disperser) were used to investigate the emission characteristics of nano-powders, including nano-titanium dioxide (nano- $\mathrm{TiO}_{2}$, primary diameter: $21 \mathrm{~nm}$ ), nano-zinc oxide (nano- $\mathrm{ZnO}$, primary diameter: $30-50 \mathrm{~nm}$ ), and nano-silicon dioxide (nano- $\mathrm{SiO}_{2}$, primary diameter: $10-30 \mathrm{~nm}$ ). A TSI SMPS (scanning mobility particle sizer), a TSI APS (aerodynamic particle sizer), and a MSP MOUDI (micro-orifice uniform deposit impactor) were used to measure the number and mass distributions of generated particles. Significant differences in specific
\end{abstract}

Special Issue Editors: Candace S.-J. Tsai,

Michael J. Ellenbecker

This article is part of the Topical Collection on

Nanotechnology, Occupational and Environmental Health

C.-J. Tsai $(\varangle)$ · G.-Y. Lin · C.-N. Liu · C.-E. He

Institute of Environmental Engineering, National Chiao

Tung University, 1001 University Road, Hsinchu 30010,

Taiwan

e-mail: cjtsai@mail.nctu.edu.tw

C.-W. Chen

Institute of Occupational Safety and Health,

Council of Labor Affairs, Executive Yuan,

99, Lane 407, Hengke Road, Shijr, Taipei 22143,

Taiwan number and mass concentration or distributions were found among different methods and nano-powders with the most specific number and mass concentration and the smallest particles being generated by the most energetic SSPD, followed by VS and RD. Near unimodal number or mass distributions were observed for the SSPD while bi-modal number or mass distributions existed for nano-powders except nano- $\mathrm{SiO}_{2}$ which also exhibited bimodal mass distributions. The 30-min average results showed that the mass median aerodynamic diameter (MMAD) and number median diameter (NMD) of the SSPD ranged $1.1-2.1 \mu \mathrm{m}$ and $166-261 \mathrm{~nm}$, respectively, for all three nano-powders, which were smaller than those of the VS (MMAD: 3.3-6.0 $\mu \mathrm{m}$ and NMD: 156-462 nm), and the RD (MMAD: 5.2-11.2 $\mu \mathrm{m}$ and NMD: 198-479 nm). For nano-particles (electric mobility diameter $<100 \mathrm{~nm}$ ), specific mass concentrations were nearly negligible for all three nano-powders and test methods. Specific number concentrations of nano-particles were low for the RD tester but were elevated when more energetic VS and SSPD testers were used. The quantitative size and concentration data obtained in this study is useful to elucidate the field emission and personal exposure data in the future provided that particle loss in the generation system is carefully assessed.

Keywords Dustiness - Nano-particle . Powder dispersion - Agglomerate $\cdot$ Rotating drum . Nano-particle mixing 


\section{Introduction}

Dust emitted in the processes of manufacture and handling of nano-materials in nano-technologyrelated workplaces may pose health risks to workers (NIOSH 2009; Schulte et al. 2008; Tsai and Pui 2009). However, actual exposure data are not enough to assess the risks of NP exposure in the workplace. Brouwer (2010) and Brouwer et al. (2009) concluded that although previous exposure studies contributed to a better understanding of the potential for exposure to MNOs (manufactured nano-objects), quantitative assessment of exposure MNOs was not possible. A more harmonized approach for analyzing and reporting of the results was recommended (Brouwer et al. 2009). In particular, few attempts have been made in establishing the practical relationship between measured dustiness and actual dust exposure at the workplace (Heitbrink et al. 1990; Brouwer et al. 2006; Tsai et al. 2011).

Therefore, it is important to measure the propensity of nano-materials for exposure assessment, control, and mitigation purposes. Different particle generation methods were proposed for testing the dustiness of nano-materials in the ISO standard (ISO/DIS 12025, 2011) including the rotating drum dustiness tester (RD) (Mark 2005; Mark et al. 2007; Schneider and Jensen 2008; Tsai et al. 2009, 2011), the vortex shaker (VS) (Maynard et al. 2004; Ogura et al. 2007), and the dynamic methods. The RD tester described in EN15051 (CEN (European Committee for Standardization) 2006) was used by Mark et al. $(2005,2007)$ to determine the dustiness of $\mathrm{TiO}_{2}(150 \mathrm{~nm})$, nano-CeO (20-30 nm), and carbon nano-fibres (60-150 nm). High-number concentration of $1 \mu \mathrm{m}$ particles measured by APS and low concentration of nano-particles measured by SMPS were observed due to agglomeration of the dust generated from the RD. A decreasing trend for number concentration of generated particles was found in Mark et al. (2007) when the crystallite size of test nano-powder increased. This result was also found in Schneider and Jensen (2008) who used a combined single-drop and a small RD to characterize the dustiness of ten different test materials. The size distribution of the generated particles measured by FMPS and APS in Schneider and Jensen (2008) showed bimodal size distribution in which one mode was located at $0.9 \mu \mathrm{m}$ while the other was located at the size range from 100 to $220 \mathrm{~nm}$. Similar bimodal size distribution was also observed in Maynard et al. (2004) and Tsai et al. (2009). Maynard et al. (2004) used VS tester to determine the dustiness of ablation SWCNT and HiPCO SWCNT. The SMPS and APS were used to determine the particle number concentration and size distribution of aerosols, respectively. The ablation SWCNT was found to be too compacted to generate aerosol. For HiPCO SWCNT, the number concentration of generated fine and nano-size particles increased with increasing agitation level, while no remarkable change was observed in the size distribution. Based on the technique by Maynard et al. (2004); Ogura et al. (2007) used the VS to conduct dustiness test and the experimental results also showed no obvious changes in the particle-size distribution of aerosols regardless of the level of agitation.

The above researches all focused on measuring the number concentrations and distributions of generated particles from test nano-materials. For exposure assessment, the mass concentration of aerosols should also be taken into consideration as is evident from the recent published mass-based recommended exposure limits for nano-sized and fine $\mathrm{TiO}_{2}$ (NIOSH 2011). Tsai et al. $(2009,2011)$ used a standard RD with a modified sampling train to obtain the number and mass distributions for nano-materials and compared the distributions obtained in the field exposure study. Results indicated that RD was able to generate similar distributions as those in the workplace. Both field and laboratory data showed that NP number and mass concentrations of manufactured materials were close to the background level. Number concentration was elevated only for particles with the electrical mobility diameter larger than $100 \mathrm{~nm}$ during bagging or feeding processes. Large fraction of nano-materials was found in the RPM (respirable particle mass) due to the agglomeration of nano-materials or attachment of nano-materials to larger particles. RPM concentration measurements were suggested to be important for the exposure assessment of nano-particles in workplace. Similar suggestion was made by Schneider and Jensen (2009).

However, in previous studies, no comparisons have been made between different generation methods and quantitative-specific particle concentrations (or the concentrations of generated particles per unit mass of dispersed nano-materials) have rarely been obtained, in particular the mass distributions. The latter is very important as nano-particles tend to become 
agglomerates in the micron-sized range which can also deposit in the alveolar region of the lung and cause health hazards. In this study, the dustiness of nano$\mathrm{TiO}_{2},-\mathrm{ZnO}$, and $-\mathrm{SiO}_{2}$ were characterized by using the standard RD, VS, and SSPD testers. The SMPS (TSI model 3936), the APS (TSI model 3321), and the MOUDI (MSP model 110) were used to obtain the number and mass distributions simultaneously for the generated particles. The specific number and mass concentration distributions were calculated and the results were compared among three generation methods.

\section{Materials and methods}

Nano- $\mathrm{TiO}_{2}$ (Degussa AEROXITE $\mathrm{TiO}_{2} \mathrm{P} 25$ ), -ZnO (Yong-Zhen, QF-Zn(w)-30, Taiwan), and $-\mathrm{SiO}_{2}$ (Degussa SIPERNAT 22S) nano-powders with the apparent bulk density of $0.13 \mathrm{~g} / \mathrm{cm}^{3}$ for nano- $\mathrm{TiO}_{2}, 0.35 \mathrm{~g} /$ $\mathrm{cm}^{3}$ for nano- $\mathrm{ZnO}$, and $0.056 \mathrm{~g} / \mathrm{cm}^{3}$ nano- $\mathrm{SiO}_{2}$ were used as the test nano-materials, which were dried in an oven controlled at $105{ }^{\circ} \mathrm{C}$ for $2 \mathrm{~h}$ before the tests. The specification of each test nano-powder is shown in Table 1.

The RD-, VS-, and SSPD-testers were used to aerosolize and de-agglomerate the tested nano-powders. The experimental set up for the RD tester is similar to that described in Tsai et al. (2009), as shown in Fig. 1a, except only two 20 ppi (pores per inch) porous foams were placed in the filter holder. Therefore, only particles smaller than the thoracic fraction were tested in the sampling train consisting of a MOUDI, an APS and a SMPS.
The VS tester was set up based on that in Ogura et al. (2007) as shown in Fig. 1b. $1 \mathrm{~cm}^{3}$ of each test nano-powder was first placed in a $70 \mathrm{~mL}$ test tube and agitated by using the VS (Vortex Genie 2, SI Inc., USA). $5 \mathrm{~L} / \mathrm{min}$ of HEPA-filtered air was used to deliver the airborne particles generated from the test nano-powder during agitation to the sampling train. An aerosol neutralizer (Model 3012, TSI Inc., St. Paul, MN, USA) was used to neutralize the generated particles in the VS and SSPD testers.

The experimental setup for the SSPD sharing the same tubing and connections with the VS is also shown in Fig. 1b. The SSPD comprising of a venturi aspirator, a capillary delivery tube, and an aluminum disk was used as the dynamic dust generation method. The test nano-powder was first loaded onto one of three concentric $\mathrm{v}$-shaped grooves of $0.66 \mathrm{~mm}$ in depth and $1.143 \mathrm{~mm}$ in width on the surface of aluminum disk, and then drawn into the capillary delivery tube by means of the venturi aspirator with $18.5 \mathrm{~L} / \mathrm{min}$ of air flow rate. The entrained particles were de-agglomerated in the venturi throat and delivered to the sampling train.

The dispersed particles were sampled by the MOUDI for $2 \mathrm{~min}$ in the RD and $30 \mathrm{~min}$ for the VS and the SSPD to determine the mass distributions and monitored by APS and SMPS for $30 \mathrm{~min}$ for the number distributions. The tests were initiated after particle concentration was reduced below 10 (SMPS) and $1 \# / \mathrm{cm}^{3}$ (APS) by drawing clean air through the testers.

Particle loss may occur inside the dustiness testers and in the tubing system which causes uncertainty when the specific concentrations are calculated.

Table 1 Specifications of the test nano-materials

\begin{tabular}{|c|c|c|c|c|c|}
\hline Nano-material & $\begin{array}{l}\text { Manufacturer } \\
\text { and model }\end{array}$ & Primary size & Bulk density & $\begin{array}{l}\text { Crystallite } \\
\text { density }\end{array}$ & $\begin{array}{l}\text { Amount of powder used } \\
\text { in the experiment (mg) }\end{array}$ \\
\hline $\mathrm{TiO}_{2}$, nano & $\begin{array}{l}\left.\text { Degussa, AEROXIDE }{ }^{(}\right) \\
\mathrm{TiO}_{2} \text { P25 }\end{array}$ & $21 \mathrm{~nm}$ & $0.13 \mathrm{~g} / \mathrm{cm}^{3} \pm 0.004 \mathrm{~g}$ & $3.95 \mathrm{~g} / \mathrm{cm}^{3}$ & $\begin{array}{l}4.5 \mathrm{~g} \pm 1 \mathrm{mg} \text { for } \mathrm{RD} \\
130 \mathrm{mg} \pm 1 \mathrm{mg} \text { for } \mathrm{VS} \\
4.3 \mathrm{mg} \pm 1 \mathrm{mg} \text { for } \mathrm{SSPD}\end{array}$ \\
\hline $\mathrm{ZnO}$, nano & $\begin{array}{l}\text { Taiwan, Yong-Zhen, QF- } \\
\text { Zn(w)-30 }\end{array}$ & $30-50 \mathrm{~nm}$ & $0.35 \mathrm{~g} / \mathrm{cm}^{3} \pm 0.01 \mathrm{~g}$ & $5.61 \mathrm{~g} / \mathrm{cm}^{3}$ & $\begin{array}{l}12.3 \mathrm{~g} \pm 1 \mathrm{mg} \text { for } \mathrm{RD} \\
350 \mathrm{mg} \pm 1 \mathrm{mg} \text { for } \mathrm{VS} \\
11 \mathrm{mg} \pm 1 \mathrm{mg} \text { for } \mathrm{SSPD}\end{array}$ \\
\hline $\mathrm{SiO}_{2}$, nano & $\begin{array}{l}\text { Degussa, SIPERNAT }{ }^{\circledR} \\
22 \mathrm{~S}\end{array}$ & $10-30 \mathrm{~nm}$ & $0.056 \mathrm{~g} / \mathrm{cm}^{3} \pm 0.001 \mathrm{~g}$ & $2.2 \mathrm{~g} / \mathrm{cm}^{3}$ & $\begin{array}{l}1.96 \mathrm{~g} \pm 1 \mathrm{mg} \text { for } \mathrm{RD} \\
56 \mathrm{mg} \pm 1 \mathrm{mg} \text { for } \mathrm{VS} \\
2.5 \mathrm{mg} \pm 1 \mathrm{mg} \text { for } \mathrm{SSPD}\end{array}$ \\
\hline
\end{tabular}


(a) Rotating drum
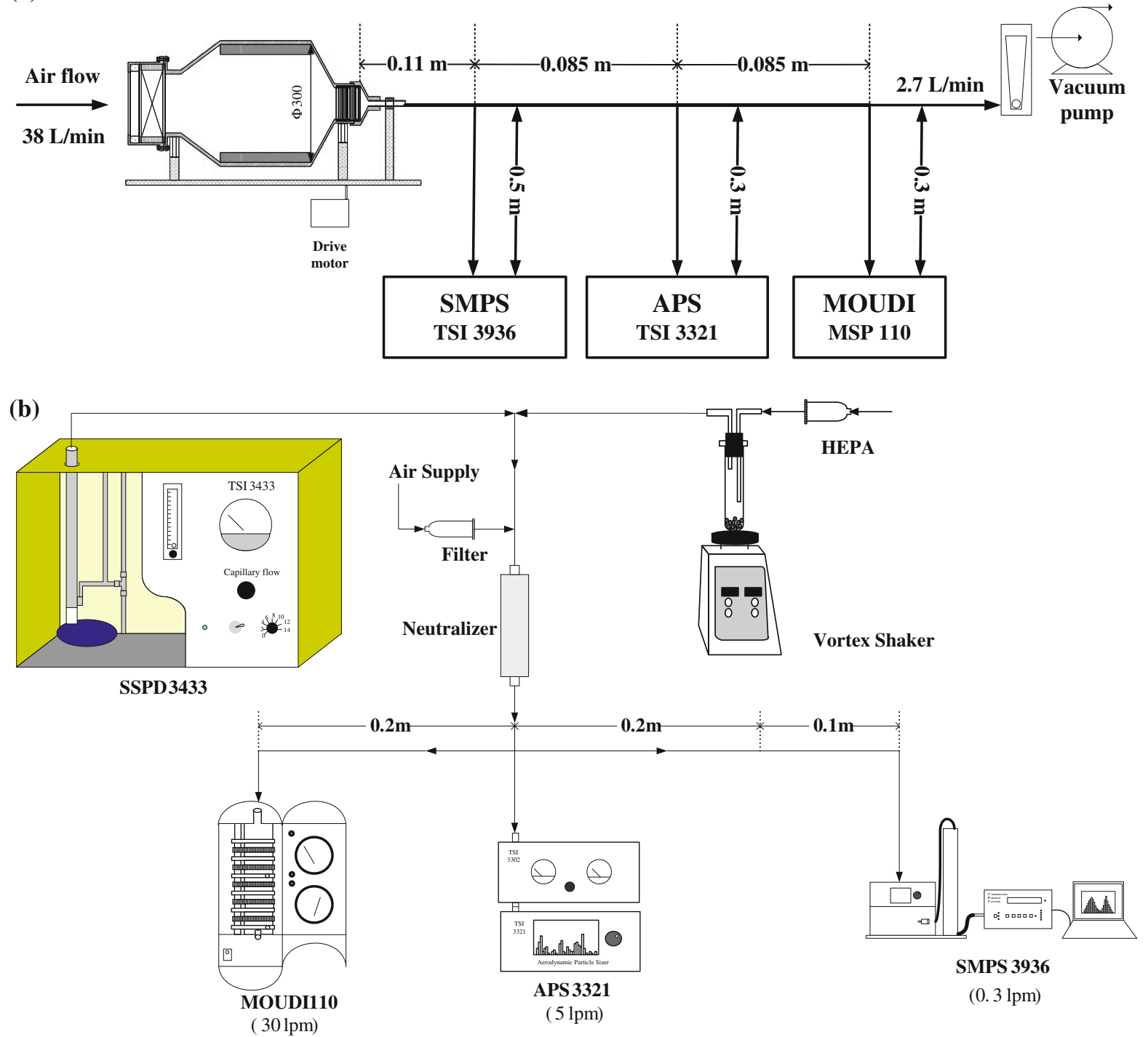

Fig. 1 Experimental setup of $\mathbf{a}$ the RD tester and $\mathbf{b}$ VS- and SSPD-testers

Particle loss mechanisms include inertial deposition at flow constrictions and bends, turbulent inertial deposition, gravitational settling, and diffusion deposition in pipes (Baron and Willeke 2001). In this study, the theoretical equations taken from Baron and Willeke (2001) were used to predict the particle loss in the tubing system. For the RD tester, the transport loss before sampling instruments were calculated to be $1.0 \%$ for SMPS, $1.4 \%$ for APS, and $1.8 \%$ for MOUDI for $10 \mu \mathrm{m}$ particles. For particles between $100 \mathrm{~nm}$ and $10 \mu \mathrm{m}$, the particle loss was calculated to be smaller than $1 \%$. Besides, there was no electrostatic neutralizer used in the RD system and the generated particles were fed horizontally into the sampling lines of the aerosol devices directly. Therefore, the total sampling efficiency was assumed to be $100 \%$ for the RD tester.

For the VS- and SSPD-testers, the transport loss in the sampling line was calculated to be less than $24.5 \%$ for particles in the size range of $10 \mathrm{~nm}-20 \mu \mathrm{m}$. There are no theoretical equations to predict the particle loss inside the VS and SSPD testers and the electrostatic neutralizer. During the dustiness testing, large amount of particles was observed to deposit inside the 
neutralizer, the transport tube connecting between the neutralizer and the VS and SSPD testers, in the upper section of the test tube of the VS and the venturi throat of the SSPD, respectively. Therefore, it is important to determine the total sampling efficiency $\left(\eta_{\mathrm{T}}\right)$ for calculating the specific particle concentrations, which is given by

$\eta_{\mathrm{T}}=\frac{W^{\prime}}{W} \times 100 \%$

where $W$ is the mass of nano-powder used in the dustiness tester $(\mathrm{g}), W^{\prime}(\mu \mathrm{g})$ is the mass of nanoparticles dispersed by the dustiness tester and sampled by the instruments. $W^{\prime}$ is calculated from the total particle mass collected by the MOUDI as

$W^{\prime}=\frac{\sum_{j=1}^{9} \Delta m_{j} \times D F \times q_{1}}{q_{2}}$

where $\Delta m_{j}$ is the mass concentration of particle sampled by each stage of the MOUDI $(\mu \mathrm{g})$; DF is the dilution factor (2.45 for the SSPD and 7.06 for the $\mathrm{VS}) ; q_{1}$ is the outlet air flow rate of the SSPD $(18.5 \mathrm{~L} / \mathrm{min})$ or the VS $(5 \mathrm{~L} / \mathrm{min}) ; q_{2}$ is the sampling flow rate of the MOUDI, $30 \mathrm{~L} / \mathrm{min}$. The $\eta_{\mathrm{T}}$ for each test nano-powder in the SSPD and VS are shown in Table 2. For each nano-powder, $\eta_{\mathrm{T}}$ of the SSPD tester was higher than that of the VS tester because the former has a higher dispersion energy than the latter, which will be discussed in the "Results and discussion" section.

The specific number $\left(\# / \mathrm{cm}^{3} \mathrm{~g}\right)$ and mass concentrations $\left(\mu \mathrm{g} / \mathrm{m}^{3} \mathrm{~g}\right)$ were calculated as

$C^{\prime}=\frac{C}{W \times \eta_{\mathrm{T}}}$

where $C$ is the generated particle number $\left(\# / \mathrm{cm}^{3}\right)$ or mass $\left(\mu \mathrm{g} / \mathrm{cm}^{3}\right)$ concentration.

\section{Results and discussion}

The RD test

The $30 \mathrm{~min}$ average specific number concentration distributions measured in the RD dustiness test for three generated particles are shown in Fig. 2. All test data show bimodal distributions, which is similar to the experimental results described in previous researches (Maynard et al. 2004; Schneider and Jensen 2008; Tsai et al. 2009). For nano- $\mathrm{TiO}_{2}$ and nano- $\mathrm{ZnO}$, the specific number concentrations of particles below $100 \mathrm{~nm}$ measured by SMPS were lower than $10 \# / \mathrm{cm}^{3} \mathrm{~g}$, which suggested that nearly no nano-particles were generated during 30 min test. Table 3 a shows the NMD, NMAD, and GSD of three generated particles at the sampling time of 2 and 30 min during the RD dustiness test. After 30 min test, the NMD and GSD were $479 \mathrm{~nm}$ and 1.89 , respectively, for $\mathrm{TiO}_{2}$ and $412 \mathrm{~nm}$ and 1.75 , respectively, for nano- $\mathrm{ZnO}$. For nano-SiO 2 , the specific number concentration distribution had NMD and GSD of $198 \mathrm{~nm}$ and 2.28, respectively. No obvious changes of NMD, NMAD, and GSD were observed suggesting that particle-size distributions were stable during 30 min sampling time. The generated nano- $\mathrm{SiO}_{2}$ particles are smaller than those from nano- $\mathrm{TiO}_{2}$ and $-\mathrm{ZnO}$ nano-powders due to more fluffy structure of nano- $\mathrm{SiO}_{2}$ nano-powder as compared with $\mathrm{TiO}_{2}$ and $\mathrm{ZnO}$ nanopowders (Ibaseta et al. 2007). The APS data show similar specific number concentration distributions among three generated particles with NMAD and GSD of 1.7-1.9 $\mu \mathrm{m}$ and 1.6-1.7, respectively.

Figure $3 \mathrm{a}, \mathrm{b}$ shows the total specific number concentration versus time measured by SMPS and APS, respectively. Both SMPS and APS data show a decaying trend with test time for nano- $\mathrm{TiO}_{2},-\mathrm{ZnO}$, and $-\mathrm{SiO}_{2}$. The most significant decaying trend for the specific concentration occurred for nano- $\mathrm{ZnO}$, which

Table 2 Total sampling efficiency $\left(\eta_{\mathrm{T}}\right)$ for each test nano-powder in SSPD and VS

\begin{tabular}{|c|c|c|c|c|c|c|}
\hline & \multicolumn{3}{|l|}{ VS } & \multicolumn{3}{|l|}{ SSPD } \\
\hline & Nano- $\mathrm{TiO}_{2}$ & Nano-ZnO & Nano-SiO ${ }_{2}$ & Nano- $\mathrm{TiO}_{2}$ & Nano-ZnO & Nano-SiO ${ }_{2}$ \\
\hline$\Delta m_{j}(\mu \mathrm{g})$ & $7038.8 \pm 1207.7$ & $4909.0 \pm 1597.2$ & $582.9 \pm 165.8$ & $888.8 \pm 199.6$ & $1664.6 \pm 348.8$ & $90.8 \pm 12.4$ \\
\hline$W^{\prime}(\mu \mathrm{g})$ & $8282.3 \pm 1421.1$ & $5776.2 \pm 1879.3$ & $685.9 \pm 195.0$ & $1342.8 \pm 301.6$ & $2515.0 \pm 527.0$ & $137.2 \pm 18.7$ \\
\hline$W(\mu \mathrm{g})$ & $116967 \pm 848.5$ & $146666.7 \pm 14542.0$ & $16933.3 \pm 9165.2$ & $4290 \pm 1180$ & $11083 \pm 1040$ & $2520 \pm 997$ \\
\hline$\eta_{\mathrm{T}}(\%)$ & $7.1 \pm 1.2$ & $3.9 \pm 0.9$ & $4.0 \pm 0.9$ & $26.80 \pm 11.36$ & $23.10 \pm 6.91$ & $5.80 \pm 1.23$ \\
\hline
\end{tabular}




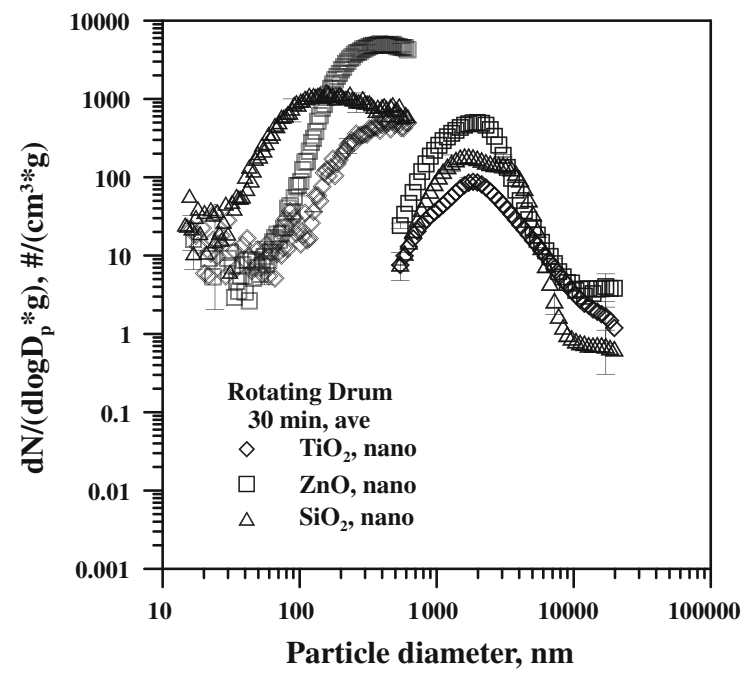

Fig. 2 The $30 \mathrm{~min}$ average specific number concentration distributions of three generated particles, the RD tester

dropped from 2593.1 to $1613.0 \# / \mathrm{cm}^{3} \mathrm{~g}$ for the SMPS data and $374.8-150.3\left(\# / \mathrm{cm}^{3} \mathrm{~g}\right)$ for the APS data during 30 min test. It is also shown in Fig. 3 that the total specific number concentration of generated particles in the RD test ranked from high to low as nano- $\mathrm{ZnO},-\mathrm{SiO}_{2}$, and $-\mathrm{TiO}_{2}$.

\section{The VS dustiness test}

Figure 4 shows the 30 min average specific number concentration distributions of aerosols generated from three test nano-powders by using the VS tester. The dustiness of three generated particles in the VS test is observed to be two orders of magnitude higher than that in the RD test. It is because the dispersion force exerted on the nano-powders in the VS tester is higher than that in the RD tester, as will be discussed later. It is noted that the average specific number concentration distributions measured by the SMPS show a lognormal distribution for nano- $\mathrm{TiO}_{2}$ and $-\mathrm{ZnO}$ with NMD and GSD of $360 \mathrm{~nm}$ and 1.86, respectively, for nano- $\mathrm{TiO}_{2}$, and $462 \mathrm{~nm}$ and 1.72 , respectively, for nano-ZnO. In comparison, the SMPS data for nano$\mathrm{SiO}_{2}$ show a random distribution since the total number concentration of aerosols was observed to decrease abruptly within the first $2 \mathrm{~min}$. As shown in Fig. 5a, the total specific number concentration of nano- $\mathrm{SiO}_{2}$ decreased abruptly from $1.99 \times 10^{5}$ to $7.38 \times 10^{4} \mathrm{\#} / \mathrm{cm}^{3} \mathrm{~g}$ from 0 to $5 \mathrm{~min}$ and fluctuated very much during the rest of the $30 \mathrm{~min}$ test. It can be attributed to the fluffy nano- $\mathrm{SiO}_{2}$ structure with much smaller apparent density as compared to that of nano$\mathrm{TiO}_{2}$ and $-\mathrm{ZnO}$ (Table 1). Nano- $\mathrm{SiO}_{2}$ nano-powder is easily dispersed as soon as the inlet air flow is first introduced into the VS tester. Subsequently, generated nano- $\mathrm{SiO}_{2}$ particles concentration drops significantly as nano- $\mathrm{SiO}_{2}$ nano-powder was lost in the upper part of the test tube leaving less nano-powder for agitation and particle generation.

The APS data show lognormal distributions for all three generated particles with the NMAD and GSD of 1.4-1.6 $\mu \mathrm{m}$ and 1.6-1.7, respectively, which are similar to the results obtained in the RD test.

Table $3 b$ shows the NMD, NMAD, and GSD of three generated particles at the sampling time of 2 and 30 min during the VS dustiness test. The NMD and GSD measured by SMPS were found to increase slightly from 331 to $360 \mathrm{~nm}$ and 1.68 to 1.86 , respectively, for nano- $\mathrm{TiO}_{2}$ and from 408 to $462 \mathrm{~nm}$ and 1.5 to 1.72 , respectively, for nano- $\mathrm{ZnO}$ with increasing time. The APS data also show a slight increasing trend for NMAD with increasing time, in which NMAD was 1621-1626 nm for $\mathrm{TiO}_{2}$ and 1392-1436 nm for $\mathrm{ZnO}$, and the GSD was 1.69-1.7 for $\mathrm{TiO}_{2}$ and 1.54-1.55 for $\mathrm{ZnO}$. These results suggested that the number concentration of nanosized or submicron particles generated from nano$\mathrm{TiO}_{2}$ and $-\mathrm{ZnO}$ nano-powders decreased as the agitation time increased. For nano- $\mathrm{SiO}_{2}$, the size distribution was unstable during the 30 min VS test.

Figure $5 \mathrm{a}, \mathrm{b}$ shows the total specific number concentration as a function of agitation time measured by SMPS and APS, respectively. Both SMPS and APS data show that the total specific number concentration of three generated particles decreased with increasing agitation time. These results are similar to that obtained in the RD dustiness test.

\section{The SSPD dustiness test}

Figure 6 shows the 30 min average specific number concentration distributions of aerosols generated from three test nano-powders by using the SSPD tester. The combined SMPS and APS data show nearly uni-modal lognormal distributions for all three generated particles, which are very much different from those of the RD and VS dustiness tests. For nano- $\mathrm{TiO}_{2}$, higher particle concentration in the size range of $30-1000 \mathrm{~nm}$ was generated compared to that of nano- $\mathrm{ZnO}$ and $-\mathrm{SiO}_{2}$. 
Table 3 NMD, NMAD, and GSD for three generated particles at the sampling time of 2 and 30 min during the (a) RD dustiness test, (b) the VS test, and (c) the SSPD test

\begin{tabular}{|c|c|c|c|c|c|c|c|c|}
\hline \multirow{3}{*}{$\begin{array}{l}\text { Rotating } \\
\text { Drum }\end{array}$} & \multicolumn{4}{|l|}{$0-2 \mathrm{~min}$, ave } & \multicolumn{4}{|c|}{ All $30 \mathrm{~min}$, ave } \\
\hline & \multicolumn{2}{|l|}{ SMPS } & \multicolumn{2}{|l|}{ APS } & \multicolumn{2}{|l|}{ SMPS } & \multicolumn{2}{|l|}{ APS } \\
\hline & NMD (nm) & GSD & $\begin{array}{l}\text { NMAD } \\
(\mathrm{nm})\end{array}$ & GSD & $\begin{array}{l}\text { NMD } \\
(\mathrm{nm})\end{array}$ & GSD & $\begin{array}{l}\text { NMAD } \\
(\mathrm{nm})\end{array}$ & GSD \\
\hline \multicolumn{9}{|l|}{$\mathrm{a}$} \\
\hline $\mathrm{TiO}_{2}$, nano & $422 \pm 29$ & $1.56 \pm 0.11$ & $1800 \pm 99$ & $1.7 \pm 0.11$ & $479 \pm 27$ & $1.89 \pm 0.05$ & $1840 \pm 47$ & $1.69 \pm 0.1$ \\
\hline $\mathrm{ZnO}$, nano & $410 \pm 38$ & $1.67 \pm 0.05$ & $1833 \pm 10$ & $1.54 \pm 0.003$ & $412 \pm 22$ & $1.75 \pm 0.004$ & $1740 \pm 2$ & $1.55 \pm 0.001$ \\
\hline $\mathrm{SiO}_{2}$, nano & $194 \pm 40.4$ & $2.3 \pm 0.21$ & $1936 \pm 35$ & $1.75 \pm 0.02$ & $198 \pm 32$ & $2.28 \pm 0.16$ & $1943 \pm 29$ & $1.74 \pm 0.01$ \\
\hline \multirow[t]{3}{*}{ Vortex shaker } & \multicolumn{4}{|l|}{$0-2 \mathrm{~min}$, ave } & \multicolumn{4}{|c|}{ All $30 \mathrm{~min}$, ave } \\
\hline & \multicolumn{2}{|l|}{ SMPS } & \multicolumn{2}{|l|}{ APS } & \multicolumn{2}{|l|}{ SMPS } & \multicolumn{2}{|l|}{ APS } \\
\hline & NMD (nm) & GSD & NMAD (nm) & GSD & NMD (nm) & GSD & NMAD (nm) & GSD \\
\hline \multicolumn{9}{|l|}{ b } \\
\hline $\mathrm{TiO}_{2}$, nano & $331 \pm 81$ & $1.68 \pm 0.03$ & $1621 \pm 6$ & $1.70 \pm 0.01$ & $360 \pm 62$ & $1.86 \pm 0.03$ & $1626 \pm 11$ & $1.69 \pm 0.001$ \\
\hline $\mathrm{ZnO}$, nano & $408 \pm 49$ & $1.5 \pm 0.25$ & $1392 \pm 82$ & $1.58 \pm 0.01$ & $462 \pm 35$ & $1.72 \pm 0.18$ & $1436 \pm 26$ & $1.58 \pm 0.01$ \\
\hline $\mathrm{SiO}_{2}$, nano & $133 \pm 43$ & $2.49 \pm 1.72$ & $1565 \pm 132$ & $1.61 \pm 0.06$ & NA & NA & $1478 \pm 98$ & $1.69 \pm 0.06$ \\
\hline \multirow[t]{3}{*}{ Vortex shaker } & \multicolumn{4}{|l|}{$0-2 \mathrm{~min}$, ave } & \multicolumn{4}{|c|}{ All $30 \mathrm{~min}$, ave } \\
\hline & \multicolumn{2}{|l|}{ SMPS } & \multicolumn{2}{|l|}{ APS } & \multicolumn{2}{|l|}{ SMPS } & \multicolumn{2}{|l|}{ APS } \\
\hline & NMD (nm) & GSD & NMAD (nm) & GSD & $\begin{array}{l}\text { NMD } \\
(\mathrm{nm})\end{array}$ & GSD & $\begin{array}{l}\text { NMAD } \\
(\mathrm{nm})\end{array}$ & GSD \\
\hline \multicolumn{9}{|l|}{ c } \\
\hline $\mathrm{TiO}_{2}$, nano & $260 \pm 68$ & $2.25 \pm 1.36$ & $547 \pm 9$ & $1.47 \pm 0.03$ & $233 \pm 48$ & $2.22 \pm 0.44$ & $561 \pm 4$ & $1.41 \pm 0.002$ \\
\hline $\mathrm{ZnO}$, nano & $276 \pm 51$ & $1.76 \pm 0.29$ & $753 \pm 3$ & $1.39 \pm 0.002$ & $261 \pm 4$ & $1.62 \pm 0.12$ & $755 \pm 7$ & $1.38 \pm 0.004$ \\
\hline $\mathrm{SiO}_{2}$, nano & $125 \pm 20$ & $1.90 \pm 0.88$ & $751 \pm 13$ & $1.5 \pm 0.09$ & $166 \pm 26$ & $2.26 \pm 0.13$ & $712 \pm 16$ & $1.42 \pm 0.06$ \\
\hline
\end{tabular}

The NMD and GSD was measured to be $225 \mathrm{~nm}$ and 2.13 , respectively, for $\mathrm{TiO}_{2}, 305 \mathrm{~nm}$ and 1.98, respectively, for $\mathrm{ZnO}$ and $164 \mathrm{~nm}$ and 2.22, respectively, for $\mathrm{SiO}_{2}$.

Table 3c shows the NMD, NMAD, and GSD of three generated particles at the sampling time of 2 and 30 min during the SSPD dustiness test. The NMD and NMAD were quite stable during the 30 min dustiness test. The average NMD and GSD in the range of 164-305 $\mathrm{nm}$ and 1.60-2.20, respectively, and NMAD and GSD in the range of 561-755 nm and 1.38-1.42, respectively, are smaller than those of the RD and VS dustiness test. Figure 7a, b shows the total specific number concentrations as a function of test time measured by the SMPS and APS, respectively. Both SMPS and APS data show relative stable particle concentrations with test time since nano-powder was carefully distributed evenly on the groove of the disk and the nano-powder was aspired completely by the capillary tube of the SSPD.

Comparison of three dustiness testers

The time-average specific mass concentration distributions of the aerosols generated by three dustiness testers are shown in Fig. 8a for the RD, Fig. 8b for the VS and Fig. 8c for the SSPD. The total specific mass concentrations were ranked from high to low as the SSPD, VS, and RD with nano-particle mass concentrations (diameter less than $100 \mathrm{~nm}$ ) being very small for all testers. The MMAD of the SSPD of three generated particles falls between 1.1 and $2.2 \mu \mathrm{m}$, which is smaller than 3.3-6.0 $\mu \mathrm{m}$ for the VS and 5.2-11.2 $\mu \mathrm{m}$ for the RD, as shown in Table 4. The first mode MMADs of the VS and RD for the lightest nano- $\mathrm{SiO}_{2}$ with the apparent density of $0.056 \mathrm{~g} / \mathrm{cm}^{3}$, 

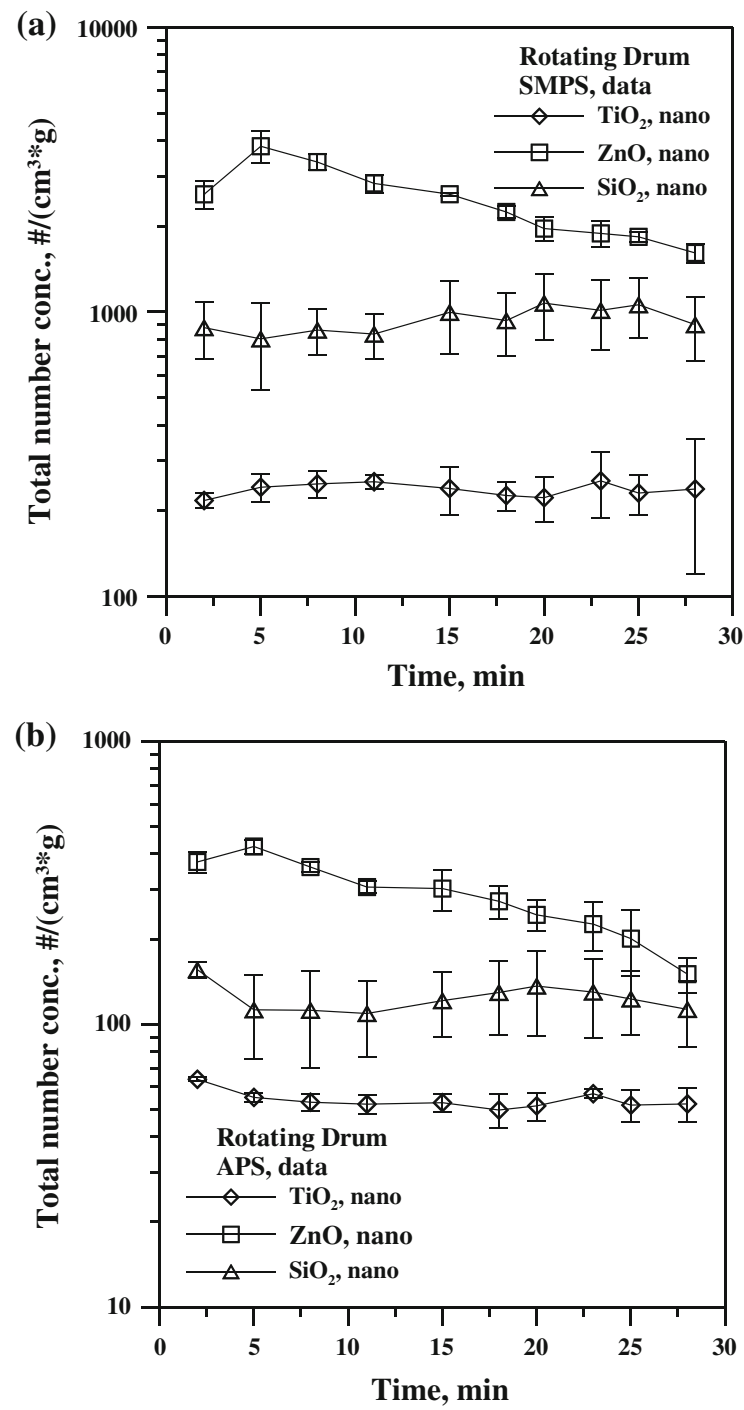

Fig. 3 Total specific number concentrations versus test time measured by a SMPS and b APS, the RD tester

1.9 and $1.8 \mu \mathrm{m}$, were quite similar to that of the SSPD, $2.1 \mu \mathrm{m}$.

For comparison purpose, the MMAD and GSD for nano- $\mathrm{TiO}_{2}$ obtained by Tsai et al. (2009) by using the $\mathrm{RD}$ dustiness tester are also listed in Table 4. The MMAD of $4.45 \mu \mathrm{m}$ obtained by Tsai et al. (2009) is shown to be smaller than MMAD of 6.45 obtained in this study. It is because only respirable particles were sample by the MOUDI in Tsai et al. (2009), while in this study both thoracic and respirable particles were allowed to be sampled by the MOUDI.

The SSPD was found to generate the highest number concentration of aerosols, followed by the

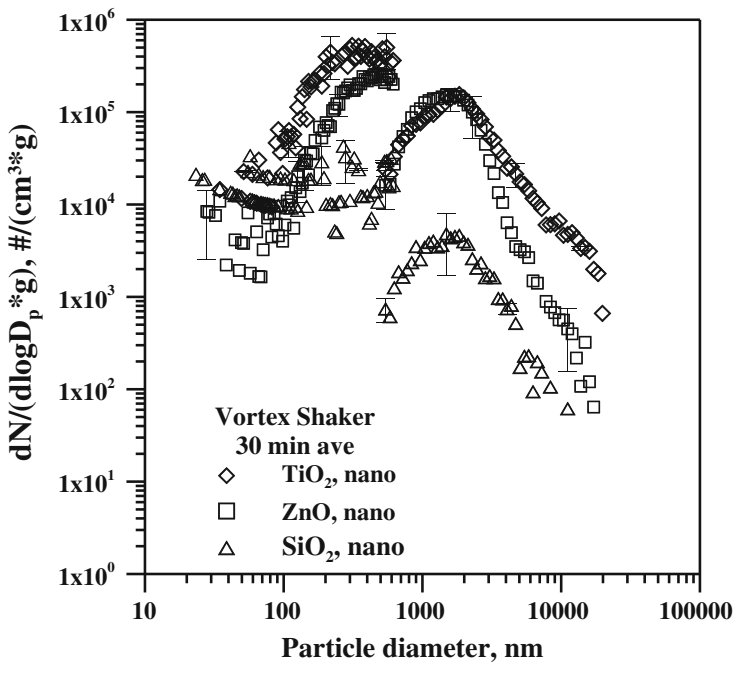

Fig. 4 The $30 \mathrm{~min}$ average specific number concentration distributions of three generated particles, the VS tester

VS and RD. It is because the SSPD has the highest dispersion energy among three testers, in which the particles are de-agglomerated by shear force generated between the high-velocity gas of $230 \mathrm{~m} / \mathrm{s}$ at the venturi throat and the lower velocity aerosol stream of $32.5 \mathrm{~m} / \mathrm{s}$ exiting the capillary tube. In comparison, both gas velocities of $0.81 \mathrm{~m} / \mathrm{s}$ for the VS and $0.001 \mathrm{~m} / \mathrm{s}$ for the RD are lower than that in the SSPD, resulting in smaller drag force for particle dispersion. The drag force for particles in the porous medium $\left(F_{\mathrm{d} \text {,porous }}\right)$ is calculated based on the Kozeny and Carman equation, which is shown as follows (Chen and Tsai 1998)

$F_{\mathrm{d}, \text { porous }}=\frac{3 \pi \mu d_{\mathrm{p}} v_{\mathrm{r}}}{C_{\mathrm{c}}} \times \frac{2 \kappa(1-\varepsilon)}{\varepsilon^{3}}$

where $\mu$ is the gas viscosity ( $\mathrm{Pa} \mathrm{s}$ ), $d_{\mathrm{p}}$ is the particle diameter $(\mathrm{m}), v_{\mathrm{r}}$ is the relative velocity between particles and gas $(\mathrm{m} / \mathrm{s}), C_{\mathrm{c}}$ is the Cunningham correction factor, $\kappa$ is an empirical constant (5.0 for irregular particles), and $\varepsilon$ is the porosity of agglomerates, which needs to be further investigated for the three test materials. At present, $\varepsilon$ is assumed to be 0.5 . Figure 9 shows the drag force on particles in three dustiness testers. As can be seen, the SSPD can produce the highest drag force on particles resulting in smaller and higher generated specific particle concentrations, followed by the VS and RD.

Figures 2, 4, and 6 show the average specific number concentrations of the generated particles 

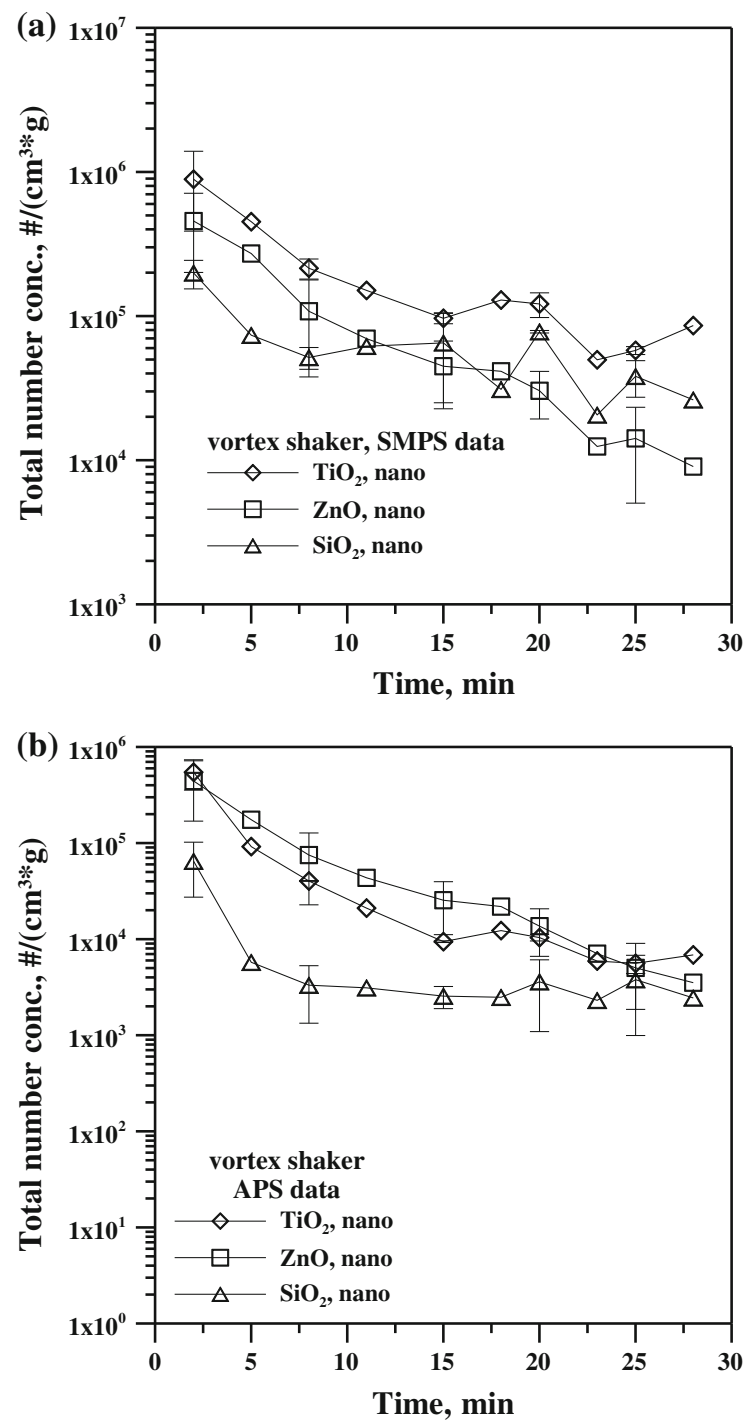

Fig. 5 Total specific number concentrations versus test time measured by a SMPS and $\mathbf{b}$ APS, the VS tester

during the $30 \mathrm{~min}$ dustiness tests as measured by the SMPS and APS, respectively. For the VS tester, the combined SMPS and APS data show that the number concentration of generated particles ranks from high to low as nano- $\mathrm{TiO}_{2}\left(3.28 \times 10^{5} \# / \mathrm{cm}^{3} \mathrm{~g}\right)$, nano- $\mathrm{ZnO}$ $\left(1.88 \times 10^{5} \mathrm{\#} / \mathrm{cm}^{3} \mathrm{~g}\right)$, and nano- $\mathrm{SiO}_{2}\left(2.83 \times 10^{4} \mathrm{\# l}\right.$ $\mathrm{cm}^{3} \mathrm{~g}$ ). The order of generated particles number concentration for the SSPD tester is observed to be the same with the VS tester as nano- $\mathrm{TiO}_{2}(2.26 \times$ $\left.10^{7} \# / \mathrm{cm}^{3} \mathrm{~g}\right)$, nano-ZnO $\left(4.75 \times 10^{6} \# / \mathrm{cm}^{3} \mathrm{~g}\right)$, and nano- $\mathrm{SiO}_{2}\left(3.77 \times 10^{6} \# / \mathrm{cm}^{3} \mathrm{~g}\right)$. For the RD tester, the nano- $\mathrm{ZnO}$ has the highest dustiness of 2,726 \#/ $\mathrm{cm}^{3} \mathrm{~g}$, followed by $\mathrm{SiO}_{2}$ of 1061.4 and $\mathrm{TiO}_{2}$ of

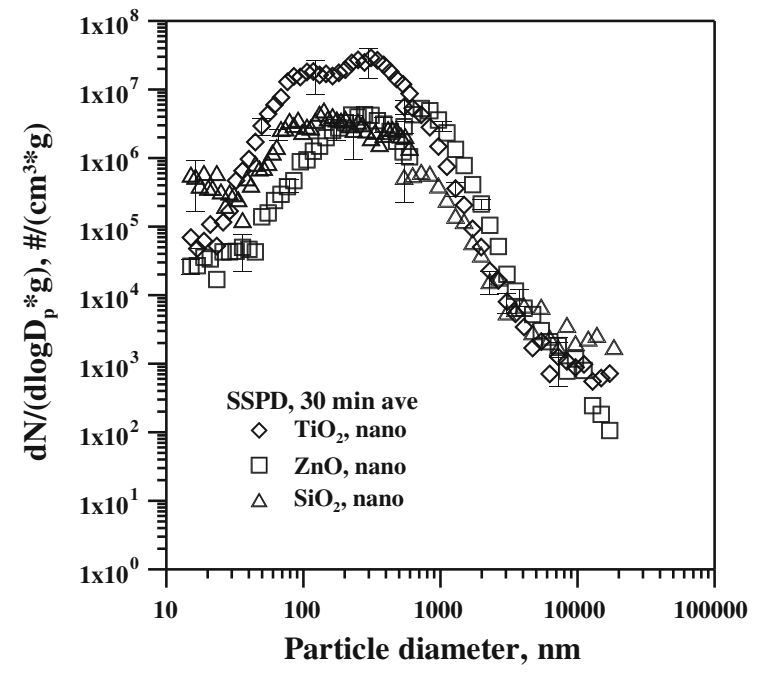

Fig. 6 The $30 \mathrm{~min}$ average specific number concentration distributions of three generated particles, the SSPD tester

$291.9 \# / \mathrm{cm}^{3} \mathrm{~g}$. That is, different test methods could result in different dustiness ranking in terms of specific particle number concentrations.

The experimental data show that nano-particles are indeed generated during dustiness tests if the dispersion energy is high enough. The RD tests show that nano-particle concentrations are low with the $30 \mathrm{~min}$ average nano-particle concentration ranking from high to low as nano- $\mathrm{SiO}_{2}\left(183.8 \# / \mathrm{cm}^{3} \mathrm{~g}\right)$, nano$\mathrm{ZnO}\left(6.7 \# / \mathrm{cm}^{3} \mathrm{~g}\right)$, and nano- $\mathrm{TiO}_{2}\left(4.8 \# / \mathrm{cm}^{3} \mathrm{~g}\right)$. The data are similar to previous study by Tsai et al. (2009, 2011). In comparison, generated nano-particle concentration increases for the VS tester and the extent of increase is substantial for the SSPD tester. The order changes as nano- $\mathrm{SiO}_{2}\left(6.4 \times 10^{3} \# / \mathrm{cm}^{3} \mathrm{~g}\right)$, nano- $\mathrm{TiO}_{2}$ $\left(5.4 \times 10^{3} \# / \mathrm{cm}^{3} \mathrm{~g}\right)$, and nano-ZnO $\left(2.1 \times 10^{3} \# / \mathrm{cm}^{3} \mathrm{~g}\right)$ for the VS tester and nano- $\mathrm{TiO}_{2}\left(2.7 \times 10^{6} \mathrm{\#} / \mathrm{cm}^{3} \mathrm{~g}\right)$, nano- $\mathrm{SiO}_{2}\left(9.5 \times 10^{5} \mathrm{\#} / \mathrm{cm}^{3} \mathrm{~g}\right)$, and nano- $\mathrm{ZnO}$ $\left(1.4 \times 10^{5} \# / \mathrm{cm}^{3} \mathrm{~g}\right)$ for the SSPD tester. These data imply that higher dispersion energy such as in the VS and SSPD could lead to nano-particle generation with high concentrations. Therefore, high-dispersion energy practices, such as cleaning by high-pressure air jet, should be avoided in workplaces.

\section{Conclusions}

In this study, the RD, VS, and SSPD testers were used to determine the dustiness of the nano- $\mathrm{TiO}_{2}$, $-\mathrm{ZnO}$, and $-\mathrm{SiO}_{2}$. The specific number and mass 

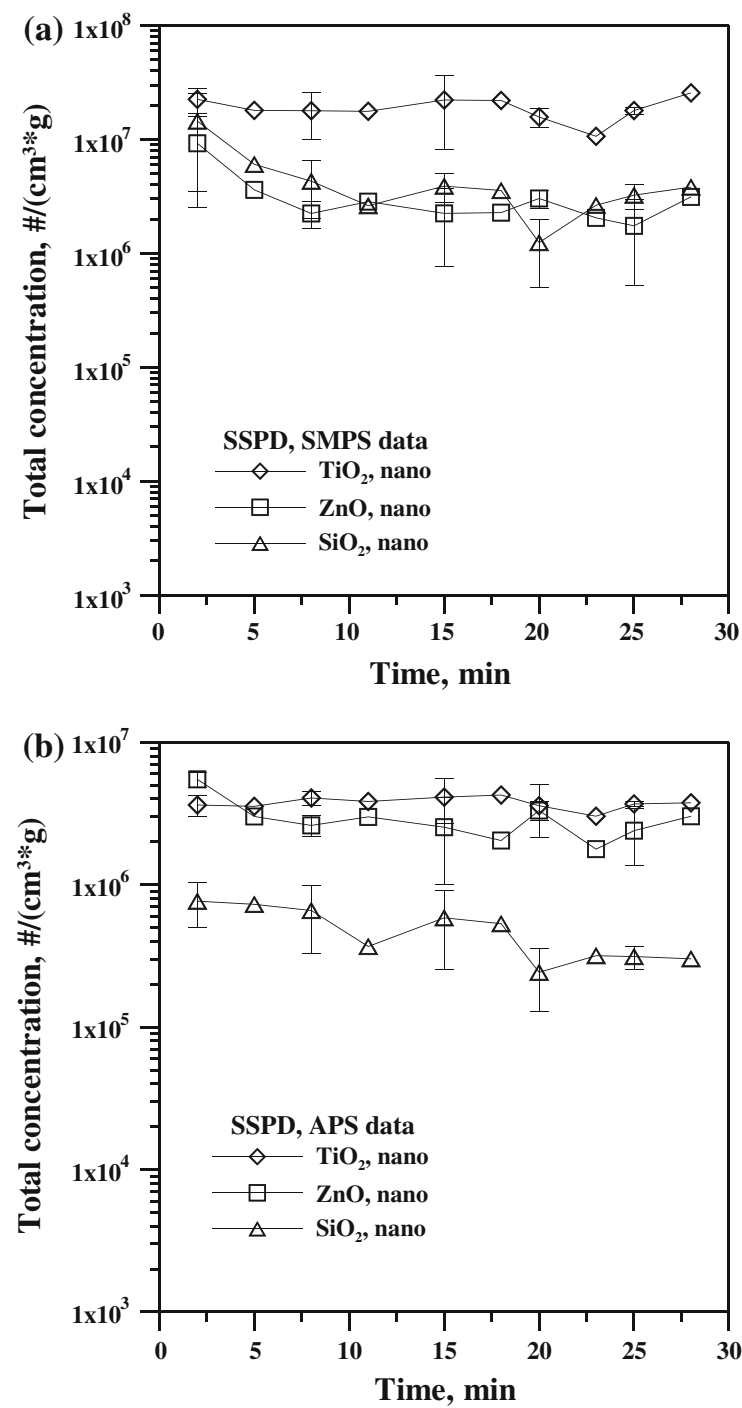

Fig. 7 Total specific number concentrations versus test time measured by a SMPS and $\mathbf{b}$ APS, the SSPD tester

concentration distributions were measured by using the SMPS, APS, and MOUDI. Results showed that different test methods could result in different specific concentrations and size distributions due to the differences in the dispersion energy and sampling efficiency of the generated particles in the test system. For the same nano-powder, different dustiness ranking in terms of specific particle number concentrations could also be different among different generation methods. In general the highest specific number and mass concentrations and the smallest particles were generated by the most energetic SSPD method, followed by the VS and RD.

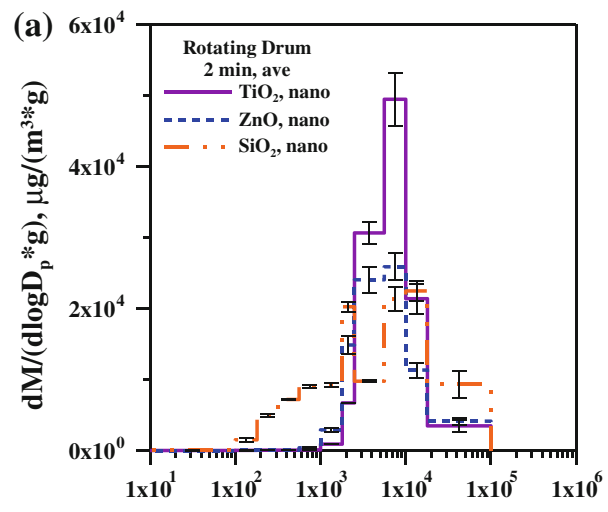

Aerodynamic diameter, $\mathrm{nm}$

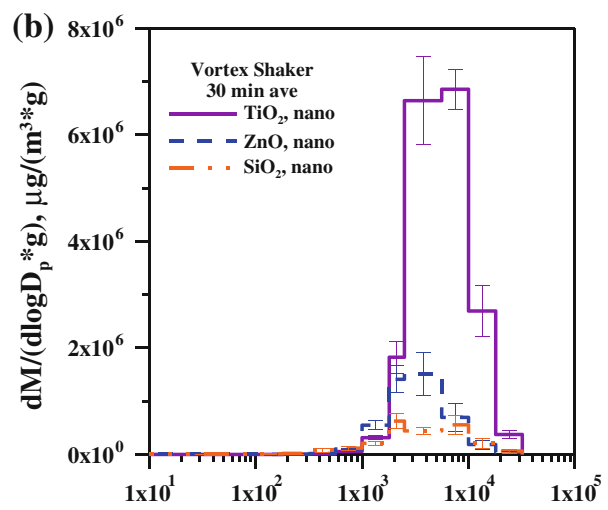

Aerodynamic diameter, $\mathrm{nm}$

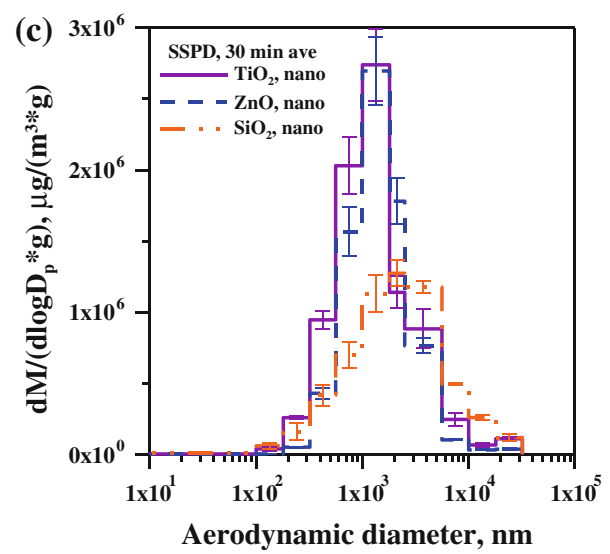

Fig. 8 Average specific mass concentration distributions of the generated particle for the three testers a RD, b VS, and c SSPD

Test results showed that total specific mass concentrations were ranked from high to low as the SSPD, VS, and RD with nano-particle mass concentrations (diameter less than $100 \mathrm{~nm}$ ) being very small for all testers. The mass median aerodynamic diameter (MMAD) and number median diameter (NMD) of the SSPD ranged 
Table 4 MMAD and GSD of generated particles for three dustiness testers

\begin{tabular}{|c|c|c|c|c|c|c|}
\hline \multirow[t]{2}{*}{ MOUDI } & \multicolumn{2}{|l|}{ SSPD } & \multicolumn{2}{|l|}{ Vortex shaker } & \multicolumn{2}{|l|}{ Rotating drum } \\
\hline & MMAD (nm) & GSD & MMAD (nm) & GSD & MMAD (nm) & GSD \\
\hline $\mathrm{TiO}_{2}$, nano & $1090 \pm 27$ & $2.11 \pm 0.05$ & $5408 \pm 129$ & $1.94 \pm 0.02$ & $\begin{array}{l}4450^{\mathrm{a}} \\
6450 \pm 100^{\mathrm{b}}\end{array}$ & $\begin{array}{l}1.83^{\mathrm{a}} \\
1.96 \pm 0.01^{\mathrm{b}}\end{array}$ \\
\hline $\mathrm{ZnO}$, nano & $1320 \pm 21$ & $1.84 \pm 0.02$ & $3280 \pm 218$ & $1.96 \pm 0.03$ & $5160 \pm 17^{\mathrm{b}}$ & $2.18 \pm 0.01^{b}$ \\
\hline $\mathrm{SiO}_{2}$, nano & $2140 \pm 375$ & $2.73 \pm 0.11$ & $\begin{array}{l}1908 \pm 73 \\
5995 \pm 174\end{array}$ & $\begin{array}{l}1.88 \pm 0.18 \\
1.97 \pm 0.02\end{array}$ & $\begin{array}{l}1847 \pm 113^{\mathrm{b}} \\
11181 \pm 1254^{\mathrm{b}}\end{array}$ & $\begin{array}{l}3.0 \pm 0.19^{\mathrm{b}} \\
2.62 \pm 0.31^{\mathrm{b}}\end{array}$ \\
\hline
\end{tabular}

a Tsai et al. (2009)

b This study

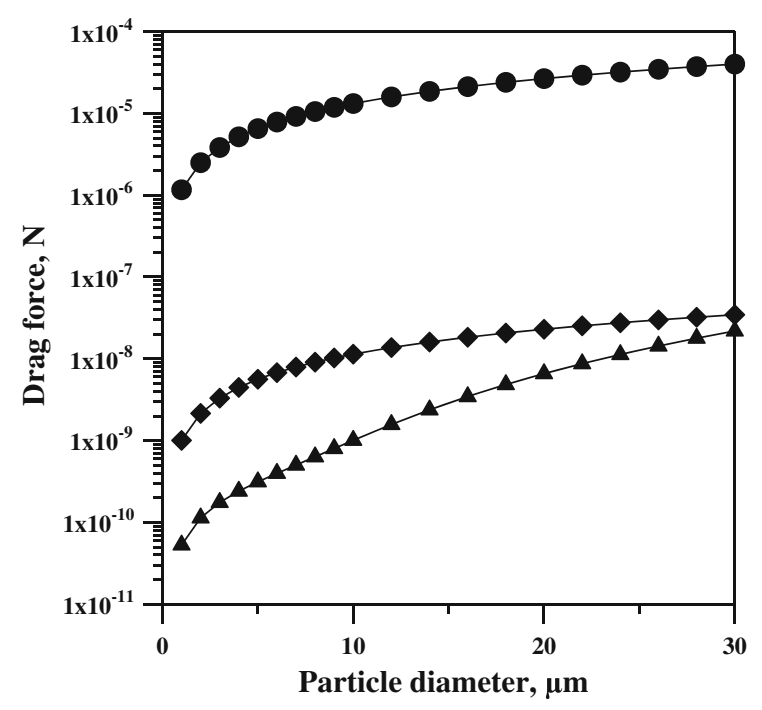

Fig. 9 Drag force on particles in the three dustiness testers

1.1-2.1 $\mu \mathrm{m}$ and 166-261 $\mathrm{nm}$, respectively, for all three nano-powders, which were smaller than those of the VS (MMAD: 3.3-6.0 $\mu \mathrm{m}$ and NMD: $156-462 \mathrm{~nm}$ ), and the RD (MMAD: 5.2-11.2 $\mu \mathrm{m}$ and NMD: 198-479 $\mathrm{nm}$ ).

The RD tests with low dispersion energy show that nano-particle concentrations are negligibly low. However, generated nano-particle concentration increases for the VS tester and the extent of increase is substantial for the SSPD tester. These data imply that higher dispersion energy such as in the VS and SSPD could lead to nano-particle generation with high concentrations. Therefore, it is recommended to use low dispersion energy to handle nano-powders or do cleaning in the workplaces.

In the future, particle loss in the VS and SSPD testers can be minimized to raise the sampling efficiency of aerosol devices so that specific particle concentrations and size distributions can be obtained with improved confidence. It is also worth conducting further tests on the influence of the amount of test materials and the electrostatic charge of the generated particles on the specific particle concentrations and size distributions.

Acknowledgments The financial support from the Taiwan Institute of Occupational Health and Safety via the contract IOSH98-H324 is gratefully acknowledged.

\section{References}

Baron PA, Willeke K (2001) Aerosol measurement: principles, techniques, and applications, 2nd edn. Wiley, New York

Brouwer DH (2010) Exposure to manufactured nanoparticle in different workplaces. Toxicology 269:120-127

Brouwer DH, Links IH, de Vreede SA, Christopher Y (2006) Size selective dustiness and exposure; simulated workplace comparisons. Ann Occup Hyg 50:452-455

Brouwer DH, van Duuren-Stuurman B, Berges M, Jankowska E, Bard D, Mark D (2009) From workplace air measurement results toward estimates of exposure? Development of a strategy to assess exposure to manufactured nano-objects. J Nanopart Res 11:1867-1881

CEN (European Committee for Standardization) (2006) Workplace atmospheres-measurement of the dustiness of bulk material-requirements and reference test methods. EN 15051:2006 (E) April 2006

Chen YH, Tsai CJ (1998) Factors influencing pressure drop through a dust cake during filtration. Aerosol Sci Technol 29:315-328

Heitbrink WA, Todd WF, Cooper TC, O'Brin DM (1990) The application of dustiness tests to the prediction of worker dust exposure. Am Ind Hyg Assoc J 51:217-223

Ibaseta N, Wistchger O, Thomas D, Biscans B (2007) Estimation of the dustiness of nanostructure powders by free falling. In: 3rd international symposium on nanotechnology, occupational and environmental health, Taipei, 29 Aug-1 Sep 2007

ISO 12025 (2011) Nanomaterials-quantification of nanoobject release from powders by generation of aerosols 
Mark D (2005) The use of reliable measurements of dustiness of chemicals in selecting the most appropriate dust control technology. IOHA 2005, Pilanesberg, p S2-3

Mark D, Bard D, Thorpe A, Wake D (2007) Some considerations for the measurement of the dustiness of nanopowders. In: 3rd international symposium on nanotechnology, occupational and environmental health, Taipei, 29 Aug-1 Sep 2007

Maynard AD, Baron PA, Foley M, Shvedova AA, Kisin ER, Castranova V (2004) Exposure to carbon nanotube material: aerosol release during the handling of unrefined single-walled carbon nanotube material. J Toxicol Environ Health Part A 67:87-107. doi:10.1080/152873904902 53688

NIOSH (2009) Approaches to safe nanotechnology: managing the health and safety concerns associated with engineered nanomaterials. March 2009. http://www.cdc.gov/niosh/ docs/2009-125/pdfs/2009-125.pdf. Accessed 10 Dec 2010

NIOSH (2011) Centers for Disease Control and Prevention, Department of Health and Human Services. Occupational Exposure to Titanium Dioxide, Current Intelligence Bulletin 63: 1-86

Ogura I, Sakurai H, Gamo M (2007) Dustiness testing of engineered nano-materials. In: Abstract, 3rd international symposium on nanotechnology, occupation and environmental health, Taipei, 29 Aug-1 Sep 2007

Schneider T, Jensen KA (2008) Combined single-drop and rotating drum dustiness test of fine to nanosize powders using a small drum. Ann Occup Hyg 52(1):23-34. doi: 10.1093/annhyg/mem059

Schneider T, Jensen KA (2009) Relevance of aerosol dynamics and dustiness for personal exposure to manufactured nanoparticles. J Nanopart Res 11:1637-1650

Schulte P, Geraci C, Zumwalde R, Hoover M, Kuempel E (2008) Occupational risk management of engineered nanoparticles. J Occup Eviron Hyg 5:239-249

Tsai CJ, Pui DYH (2009) Recent advances and new challenges of occupational and environmental health of nanotechnology. J Nanopart Res 11(1):1-4

Tsai CJ, Wu CH, Leu ML, Chen SC, Huang CY, Tsai PJ, Ko FH (2009) Dustiness test of nanopowders using a standard rotating drum with a modified sampling train. J Nanopart Res 11(1):121-131

Tsai CJ, Huang CY, Chen SC, Ho CE, Huang CH, Chen CW, Chang CP, Tsai PJ, Ellenbecker MJ (2011) Exposure assessment of nano-sized and respirable particles at different workplaces. J Nanopart Res 13(9):121-131 